\title{
Non-Newtonian Film Thickness Formation in Ultra-thin Film
}

\author{
M. F. Abd Al-Samieh \\ Mechanical Design \& Production Department, Military Technical College, \\ Cairo, Egypt \\ Email: mohamed.fahmy203@hotmail.com
}

\begin{abstract}
This paper aims to show the characteristics of ultra-thin films for non-Newtonian fluid using Ree-Eyring model where intermolecular forces of solvation and Van der Waal's are considered in addition to the hydrodynamic action to fulfill an identified need for such a conjunction. In this case, the film thickness and pressure distribution are obtained by simultaneous solution of the modified Reynolds' equation incorporating the effect of non-Newtonian fluid, film thickness equation including elastic deformation caused by all contributing pressures and the load balance equation using Newton-Raphson method with Gauss-Seidel iterations. Effect of changing the operating conditions of speed, load, Eyring shear stress and slide-roll ratio on the characteristic of the contact has been studied. The results show that, for the case where the hydrodynamic action is the only pressure acting to support the applied load capacity, the film thickness and the pressure gradient at the exit of the contact obtained using non-Newtonian model is different than that formed using the Newtonian model especially for the increased value of slide-roll ratio. The main results of this study are that for ultra-thin film, the film thickness formed using non-Newtonian model is smaller compared to that obtained using Newtonian case and the discretization of the film thickness as the gap is reduced occurs similar to the results obtained using Newtonian model. The pressure shape shows no difference compared to that formed using the Newtonian case in which an oscillation around the Hertizan contact pressure shape due to the solvation effect appears. The results also show that for ultra-thin film, changing the Eyring shear stress does not affect the film thickness formation.
\end{abstract}

Keywords: Newtonian; non-Newtonian, ultra-thin films, elastohydrodynamics, solvation; Van der Waal's force.

\section{NOMENCLATURE}

D Deformation influence coefficient matrix

$l \quad$ Dimensionless side leakage boundary distance

$b \quad$ Radius of Hertzian contact regio

C Constant defined in Eq. (12)

$a \quad$ Lubricant molecular diameter

$E$ ' Reduced modulus of elasticity

$G^{*} \quad$ Materials' parameter, $G^{*}=E^{\prime} \alpha$

$h \quad$ Lubricant film thickness

$\begin{array}{ll}X, Y & \begin{array}{l}\text { Dimensionless co-ordinates, } X=x / b, \\ Y=y / b\end{array} \\ U^{*} & \begin{array}{l}\text { Speed (or rolling viscosity) } \\ \text { parameter }\end{array} \\ u & \text { Speed of entraining motion } \\ u_{s} & \text { Sliding speed } \\ Z & \text { Viscosity-pressure index } \\ \alpha & \text { Pressure of viscosity coefficient } \\ \delta & \text { Total elastic deformation } \\ \varepsilon, \xi & \text { Constants used in Eq. (3) }\end{array}$




\begin{tabular}{|c|c|c|c|}
\hline$H$ & Dimensionless film thickness & $\eta$ & Lubricant dynamic viscosity \\
\hline$H_{0}$ & $\begin{array}{l}\text { Dimensionless central oil film } \\
\text { thickness }\end{array}$ & $\eta_{0}$ & $\begin{array}{l}\text { Atmospheric lubricant dynamic } \\
\text { viscosity }\end{array}$ \\
\hline$A$ & Hamaker constant & $\Omega$ & Under-relaxation factor \\
\hline$m$ & Dimensionless inlet distance & $\rho$ & Lubricant density \\
\hline$n_{x}, n_{y}$ & $\begin{array}{l}\text { Number of computational grid } \\
\text { nodes }\end{array}$ & $\rho_{\mathrm{o}}$ & Atmospheric lubricant density \\
\hline$P$ & Total contact pressure & $\bar{\rho}$ & Dimensionless lubricant density \\
\hline$p_{h}$ & Hydrodynamic pressure & $\bar{\eta}$ & Dimensionless lubricant viscosity \\
\hline$p_{s}$ & $\begin{array}{l}\text { Solvation pressure due to surfaces' } \\
\text { interaction force }\end{array}$ & $\tau_{0}$ & Eyring shear stress \\
\hline$p_{v d w}$ & $\begin{array}{l}\text { Pressure due to molecular Van der } \\
\text { Waal's force }\end{array}$ & $\tau_{\mathrm{m}}$ & $\begin{array}{l}\text { Mean shear stress for the Eyring } \\
\text { model }\end{array}$ \\
\hline$P$ & $\begin{array}{l}\text { Dimensionless total contact } \\
\text { pressure }\end{array}$ & $\begin{array}{l}\varphi_{\mathrm{x}} \\
\varphi_{\mathrm{y}}\end{array}$ & The effective viscosities \\
\hline$P_{h}$ & $\begin{array}{l}\text { Dimensionless hydrodynamic } \\
\text { pressure }\end{array}$ & $\varepsilon$ & $\begin{array}{l}\text { Dielectric constant of the liquid in } \\
\text { Eq. (13) }\end{array}$ \\
\hline$P_{H e r}$ & $\begin{array}{l}\text { Maximum Hertzian contact } \\
\text { pressure }\end{array}$ & $\varepsilon_{o}$ & $\begin{array}{l}\text { Dielectric permittivity of the free } \\
\text { space in Eq. (13) }\end{array}$ \\
\hline$P_{s}$ & Dimensionless solvation pressure & $k$ & The Debye length in Eq. (13) \\
\hline$P_{v d w}$ & $\begin{array}{l}\text { Dimensionless Van der Waals' } \\
\text { pressure }\end{array}$ & $\omega_{0}$ & Surface potential in Eq. (13) \\
\hline$N$ & Total number of mesh points & \multicolumn{2}{|c|}{ Superscripts } \\
\hline$R$ & $\begin{array}{l}\text { Reduced radius of counter formal } \\
\text { contact }\end{array}$ & $i, j$ & $\begin{array}{l}\text { Contravariant influence coefficient } \\
\text { indices }\end{array}$ \\
\hline$S R R$ & Slide-roll ratio & & Iteration index \\
\hline$K$ & Elliptical ratio & \multicolumn{2}{|c|}{ Subscripts } \\
\hline$w$ & Normal applied contact load & $k, l$ & $\begin{array}{l}\text { Covariant influence coefficient } \\
\text { indices }\end{array}$ \\
\hline$W$ & Load parameter & & \\
\hline
\end{tabular}

\section{INTRODUCTION}

The early numerical studies of elastohydrodynamic theory assume many simplifying assumptions such as the assumption of isothermal conditions and assumption of Newtonian fluid model. In real operations these assumptions fail in representing the physical reality of the contact. Several authors have studied the non-linear behavior of viscosity with shear stress and found that the lubricants behave as a non-Newtonian at high shear stresses as shown for example by Johnson [1] and Gohar and Rahnejat [2]. There have been many studies for the effect of non-Newtonian fluids on a number of mechanical elements, such as bearings [3], gears and cam follower pairs [4] with thermal effects and extremes of slide-roll ratio. In fact, many rheology models have been introduced to discover the characteristic of non-Newtonian lubricant as shown by Johnson and Tevaarwerk [5] such as the Ree-Eyring model, the power-law model, the Johnson-Tevaarwerk model and others. The Ree-Eyring model is recommended by many researchers and is applied extensively $[6,7]$ for lubrication performance studies of, for example, gears and cams.

Lalit Vashishth [8] numerically investigated the effect of changing various operating parameters such as load, speed and slide-roll ratio on coefficient of friction 
and on the formation of minimum and central film thickness in elastohydrodynamic line contacts using Ree-Eyring model. He found that the assumption of Newtonian fluids used in classical film thickness formulas results in large errors in the estimation of film thickness for the case where sliding is assumed and the Ree-Eyring model can capture the effect of increased load which leads to high shear stresses.

Liu [9] studied in detail through a line contact model the working parameters effects (load, speed, slide-roll ratio), the non-Newtonian effects, surface topography effects and the thermal effects on the formation of pressure, film thickness and friction coefficient. From his results, he showed that, in the nominal contact zone the minimum film thickness does not change significantly from the Newtonian solution to a certain value of slide-roll ratio. Ildiko' Ficza et al [10] and Ildiko' Ficza [11] have presented numerical solutions to investigate the effect of non-Newtonian fluid using Ree-Eyring model on the behavior of pressure and film thickness profiles under different rollingsliding conditions and compare their numerical simulation results with experimental measurements. They found that, the non-Newtonian model give accurate results compared to the experimental results in case when sliding condition is present.

Many experimental works for thin film lubrication reported by Guangteng and Spikes [12], Spikes [13] and Hartl et al [14] showed that the viscosity of the fluid at such thin film lubrication behaves as non-Newtonian properties. Lionel Bureau [15] studied experimentally using Surface Forces Apparatus the shear rates rheology of octamethylcyclotetrasiloxane (OMCTS) confined between two molecular level smoothness surfaces and drained into molecularly thin films. He found that OMCTS under increasing confinement shows viscosity enhancement and the dependence of the flow stress on shear rate shifts from linear relation to non-linear relation. The value of the shear stress above which the relation between the shear stress and shear strain rate of the lubricant becomes nonlinear shifts towards higher values as the confinement decreases, therefore OMCTS exhibits non-Newtonian features. Li-Ming et al [16] investigated numerically the effects of flow rheology and adsorbed layers on thin film lubrication using power-law model for circular contacts problem. They showed that the viscosity of the adsorption layer and its thickness significantly influence the lubrication characteristics of the contact conjunction in addition to the flow index of the power-law model.

Studies reported by Chan and Horn [17], Horn and Israelachvili [18] and Israelachvili [19], showed that for molecular surfaces, effects of intermolecular forces of solvation and Van der Waal's play an important role in lubricant film formation. Various numerical studies have been developed, including the effects of intermolecular force of solvation and long-range force of Van der Waal's. They include the work represented by Al-Samieh and Rahnejat [20,21], Gohar and Rahnejat [2], Chong et al [22], $\mathrm{Ku}$ et al [23] and Al-Samieh [24,25] for the study of ultra-thin film for lightly loaded contacts.

In this paper a comparison between the Newtonian and non-Newtonian fluid using Ree-Eyring model is shown for elastohydrodynamic lubrication for point contact problem. The effect of changing the operating conditions such as speed, load, Eyring shear stress and slide-roll ratio on the formation of film thickness and pressure distribution has been studied. The characteristic of ultra-thin film for lightly loaded conjunction included the non-Newtonian fluid is also studied where the effects of solvation and Van der Waal's is considered in addition to the hydrodynamic action to discuss the formation of film thickness and pressure distribution profiles that are formed between two molecularly smooth surfaces. 


\section{BACKGROUND THEORY}

In case of a non-Newtonian elastohydrodynamic problem, the Reynolds equation, the lubricant rheological model, the elastic film shape including the elastic deformation, and the load balance equation must be considered and solved simultaneously to obtain the characteristics of the lubricated contacts. In the case of ultra-thin film thickness, the total pressure $P$ can be calculated as shown by Al-Samieh and Rahnejat [20,21], Chong et al [22] and Al-Samieh [24, 25] as:

$$
P=P_{s}+P_{v d w}+P_{e}+P_{h}
$$

Where: $p_{h}$ is the hydrodynamic pressure, $p_{v d w}$ is the Van der Waal's pressure, $p_{s}$ is the solvation pressure and $p_{e}$ is the electrostatic component for the case of polar lubricant.

\section{Hydrodynamic Pressure}

The Reynolds equation can be presented for point contact steady state condition as:

$$
\frac{\partial}{\partial \mathrm{X}}\left(\frac{\bar{\rho} \mathrm{H}^{3}}{\bar{\eta}} \frac{\partial \mathrm{P}_{\mathrm{h}}}{\partial \mathrm{X}} \varphi_{\mathrm{x}}\right)+\frac{1}{\mathrm{~K}^{2}} \frac{\partial}{\partial \mathrm{Y}}\left(\frac{\bar{\rho} \mathrm{H}^{3}}{\bar{\eta}} \frac{\partial \mathrm{P}_{\mathrm{h}}}{\partial \mathrm{Y}} \varphi_{\mathrm{y}}\right)=\lambda \frac{\partial}{\partial \mathrm{X}}(\bar{\rho} \mathrm{H})
$$

Where the following dimensionless variables apply; $X=x / b, Y=y / a, \bar{\eta}=\eta / \eta_{0}, \bar{\rho}=\rho / \rho_{0}$, $H=h R_{x} / b^{2}, \quad P_{h}=p_{h} / p_{H e r}$ and $\lambda=\frac{12 u \eta_{o} R_{x}^{2}}{b^{3} P_{H e r}} \varphi_{\mathrm{x}}$ and $\varphi_{\mathrm{y}}$ are the effective viscosities or flow factors as given by Greenwood [26]. For the Ree-Eyring fluid the effective viscosities can be given as $\varphi_{\mathrm{x}}=\cosh \left(\frac{\tau_{\mathrm{m}}}{\tau_{0}}\right), \varphi_{\mathrm{y}}=\frac{\sinh \left(\tau_{\mathrm{m}} / \tau_{0}\right)}{\tau_{\mathrm{m}} / \tau_{0}}$ and $\tau_{\mathrm{m}}=\tau_{0} \sinh ^{-1}\left[\eta \mathrm{u}_{\mathrm{s}} /\left(\tau_{0} \mathrm{~h}\right)\right]$. $\tau_{0}$ represent the Eyring shear stress which is defined as the value of the shear stress above which the relation between the shear stress and shear strain rate of the lubricant becomes nonlinear and $\tau_{m}$ represent the mean shear stress for the Eyring model (see Greenwood [26]). Note that, for Newtonian lubricant, the effective viscosities $\Phi_{\mathrm{x}}=\Phi_{\mathrm{y}}=1$.

The variation of density with pressure is shown by Dowson and Higginson in 1959 as Eq. (3).

$\bar{\rho}=1+\frac{\varepsilon P_{h} P_{\text {Her }}}{1+\xi P_{h} P_{\text {Her }}}$

where, the constants $\varepsilon$ and $\zeta$ depends on the type of lubricant. The relation between the viscosity and pressure is given by Roelands in 1966 as Eq. (4).

$\bar{\eta}=\exp \left[\ln \eta_{\circ}+9.67\right]\left[\left(1+5.1 * 10^{-9} P_{h} P_{H e r}\right)^{Z}-1\right]$

where $Z=\frac{\alpha}{5.1 * 10^{-9}\left[\ln \eta^{\circ+9.67]}\right.}$. The film thickness equation can be given as Eq. (5) [2]: 


$$
H(X, Y)=H_{\circ}+\frac{(X-m)^{2}}{2}+\frac{K^{2} R_{x}}{R_{y}} \frac{(Y-l)^{2}}{2}+\frac{R_{x} \delta(X, Y)}{b^{2}}
$$

where the elastic deformation can be represented as Eq. (6).

$$
\delta_{I, J}(x, y)=\frac{2}{\Pi} \sum_{j=1}^{n y} \sum_{i=1}^{n x} P_{i, j} D_{i^{*}, j^{*}}
$$

where $i^{*}=|I-i|+1, j^{*}=|J-j|+1$

The Newton-Raphson method is applied for the solution of the Reynolds'equation in the following form [24, 25].

$$
\sum_{l=2}^{m y-1} \sum_{k=2}^{m x-1} J_{k, l}^{i, j} \Delta \bar{P}_{k, l}=-F_{i, j}
$$

where,

$$
J_{k, l}^{i, j}=\frac{\partial F_{i, j}}{\partial P_{k, l}}
$$

Using the Gauss-Seidel iteration method, Eq. (7) can be written as:

$$
\Delta P_{k, l}^{n}=\left(-F_{i, j}-J_{k-1, l}^{i, j} \Delta P_{k-1, l}^{n}-J_{k+1, l}^{i, j} \Delta P_{k+1, l}^{n-1}-J_{k, l-1}^{i, j} \Delta P_{k, l-1}^{n}-J_{k, l+1}^{i, j} \Delta P_{k, l+1}^{n-1}\right) / J_{k, l}^{i, j}
$$

The pressure can be updated using under-relaxation factor according to:

$$
P_{i, j}^{n}=P_{i . j}^{n-1}+\Omega \Delta P_{i, j}^{n}
$$

where $\Omega$ is under-relaxation factor and its value equal 0.01 . The criterion for the pressure convergence is $\left[\frac{\sum_{i} \sum_{j}\left(\bar{P}_{i, j}^{n}-\bar{P}_{i, j}^{n-1}\right)^{2}}{N}\right]^{0.5} \leq 10^{-4}$ The criterion for the load balance convergence is given as $\left|\iint P(X, Y) d X d Y-\frac{2}{3} \pi\right| \leq 10^{-4}$.

\section{Intermolecular Forces in Narrow Conjunctions}

The total pressure between two moving contacting surfaces separated by a lubricant includes the hydrodynamics pressure, solvation, Van der Waal's and double layer electrostatic pressure in case where a polar lubricant is used between the two surfaces.

Chan and Horn [17], Horn and Israelachvili [18], Canova [27], Thaicharoen and Schwarzkopf [28], Prakash [29], Morciano et al [30] and Peiyuan et al [31] showed that experimentally and theoretically the characteristic of long range force of Van der Waal's, intermolecular force of solvation and electrostatic force. Horn and Israelachvili [18] represent the Van der Waal's, solvation and electrostatic pressure as a function of distance as follows: 


$$
\begin{aligned}
& P_{v d w}=\frac{-A}{6 \pi h^{3}} \\
& P_{s}=-C e^{\frac{-h}{a}} \cos (2 \pi h / a) \\
& p_{\text {elest }}=2 \varepsilon \varepsilon_{o} \kappa^{2} \omega_{0}{ }^{2} e^{-\kappa h}
\end{aligned}
$$

\section{RESULTS AND DISCUSSION}

The lubricant, material properties and working conditions used in the current analysis are given in Table 1. Comparisons between the current numerical results and that reported by Ildiko' Ficza et al [10] and Ildiko' Ficza [11] for the Newtonian and nonNewtonian rheology of lubricant using Ree-Eyring model for the operating conditions that given in Table 1 are shown in detail in Table 2 and in Figure 1. For the nonNewtonian model, the Ildiko' Ficza et al [10] and Ildiko' Ficza [11] assumed that the slide-roll ratio (SRR) of 5\% and the Ree-Eyring model shear stress $\tau_{0}$ of $6 \mathrm{MPa}$ in their numerical solution to evaluate the mean shear stress. The Newtonian model assumed pure rolling conditions (i. e $\mathrm{SRR}=0 \%$ ). Figure 1 shows minimum film thickness variation with the rolling speed for the Newtonian and non-Newtonian lubricant for the data that shown in table (2). It is clear that, the current numerical results conform well to that published by the Ildiko' Ficza et al [10] and Ildiko' Ficza [11] for Newtonian and non-Newtonian fluids using Ree-Eyring model with slide-roll ratio of 5\%. Comparisons between the two rheology of lubricant show that almost no difference in film thickness variation with speed especially at low speed of entraining motion. Therefore, the Newtonian model can give an approximately accurate prediction of the actual film thickness between the two mating surfaces at pure rolling motion. Film thickness profile and pressure shape in the direction of entraining motion through the central film from the current numerical solutions for the Newtonian and non-Newtonian models shown in Figure 2 supports the above arguments for the case where the applied load of $27 \mathrm{~N}$ and speed of entraining motion of $0.125 \mathrm{~m} / \mathrm{sec}$ was used.

Table 1. Lubricant, material properties and working conditions.

\begin{tabular}{lll}
\hline Designation & Properties & Values \\
\hline$\eta_{o}$ & Viscosity & $0.22 \mathrm{~Pa} . \mathrm{s}$ \\
$\alpha$ & Viscosity coefficient for pressure & $24 \mathrm{GPa}^{-1}$ \\
$\varepsilon$ & Constant used in density Eq. (3) & $5.83 \times 10^{-10} \mathrm{~Pa}$ \\
$\xi$ & Constant used in density Eq. (3) & $1.68 \times 10^{-9} \mathrm{~Pa}$ \\
$\tau_{0}$ & Eyring model shear stress & $6 \mathrm{MPa}$ \\
$E$ & Reduced modulus of elasticity & $123.8 \mathrm{GPa}$ \\
$R_{x}$ & Reduced radius of curvature & $0.0127(\mathrm{~m})$ \\
$F$ & Load & $27 \mathrm{~N}$ \\
$u$ & Speed & $(0.01-0.4) \mathrm{m} / \mathrm{s}$ \\
\hline
\end{tabular}




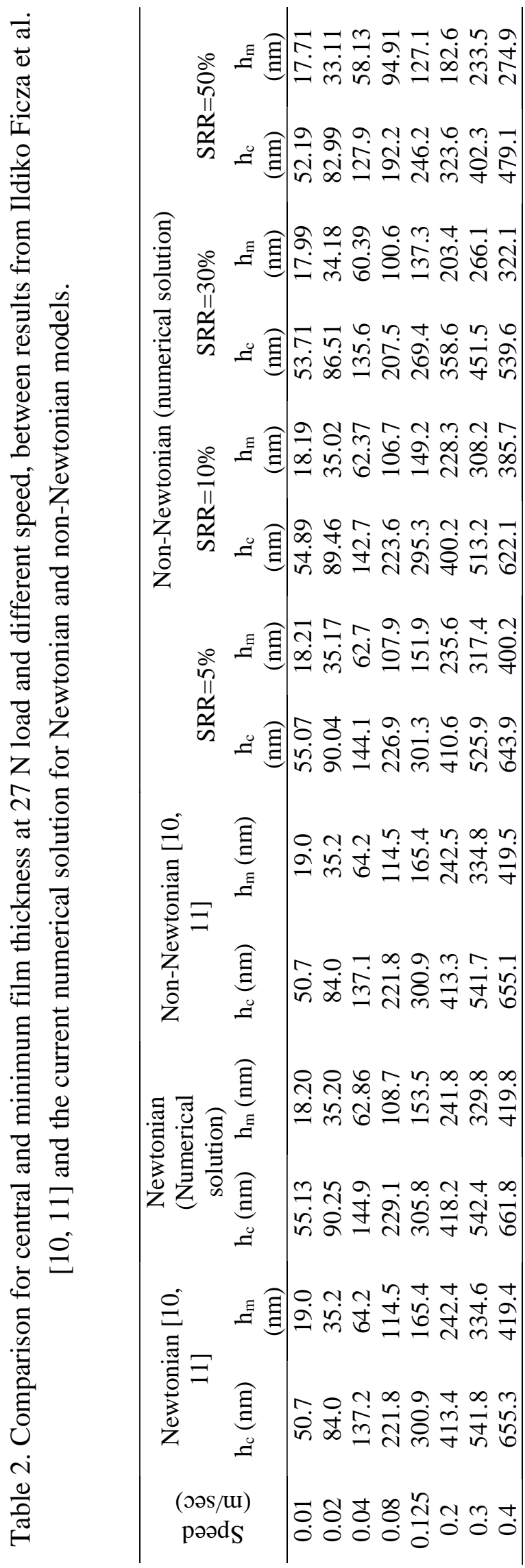


By changing the value of slide-roll ratio from $5 \%$ to $50 \%$, the difference between the two rheology models of Newtonian and non-Newtonian appears and becomes more pronounced, resulting in decreasing the minimum film thickness by increasing the value of slide-roll ratio. Effect of changing the slide-roll ratio on the film thickness is shown in detail Table 2 and in Figure 3. This figure presents the variation of minimum film thickness with speed for different values of slide-roll ratio at a constant applied load of $27 \mathrm{~N}$. At low speed of entraining motion of an approximately up to 0.1 $\mathrm{m} / \mathrm{sec}$, no change in film thickness with changing the slide-roll ratio. At speed higher than $0.1 \mathrm{~m} / \mathrm{sec}$, the film thickness decreases with increasing the slide-roll ratio.

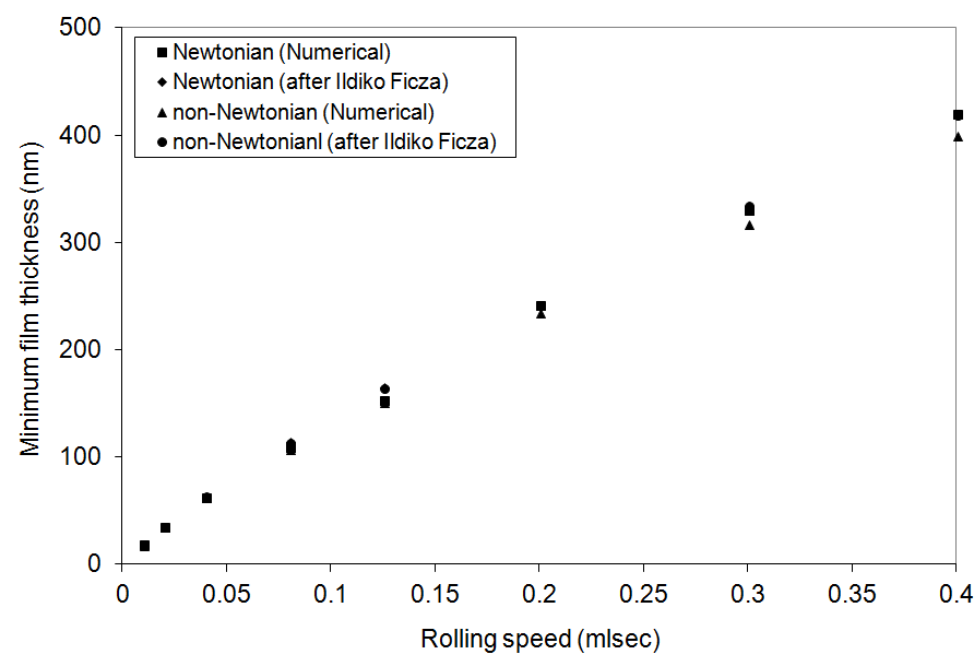

Figure 1. Minimum film thickness variation with speed for Newtonian and non-

Newtonian models at constant load of $27 \mathrm{~N}$.

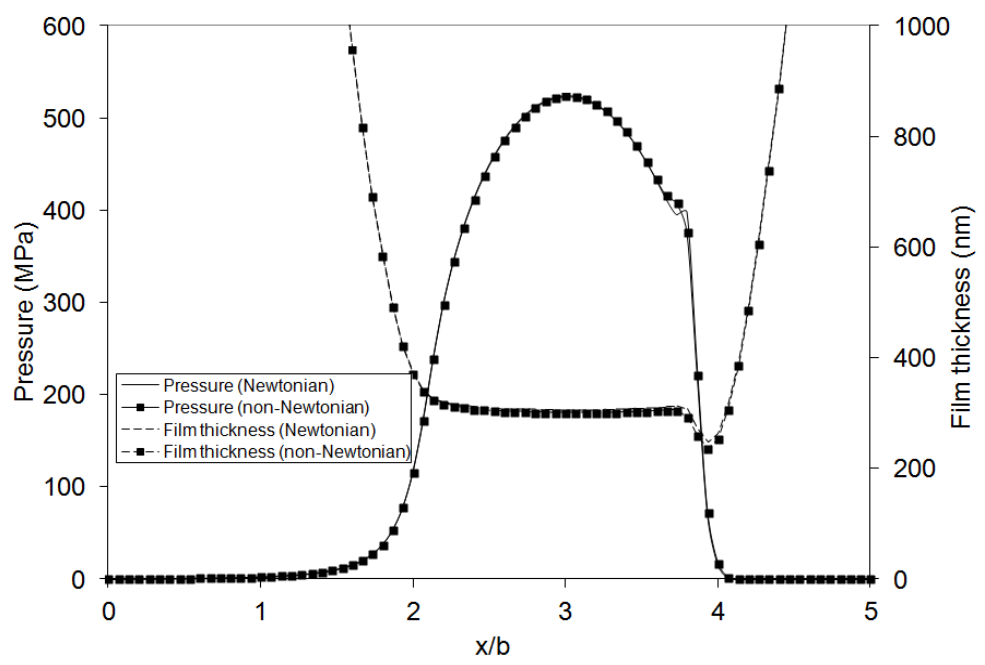

Figure 2. Pressure profile and film thickness for Newtonian and non-Newtonian models for load of $27 \mathrm{~N}$ and speed of $0.125 \mathrm{~m} / \mathrm{sec}$. 


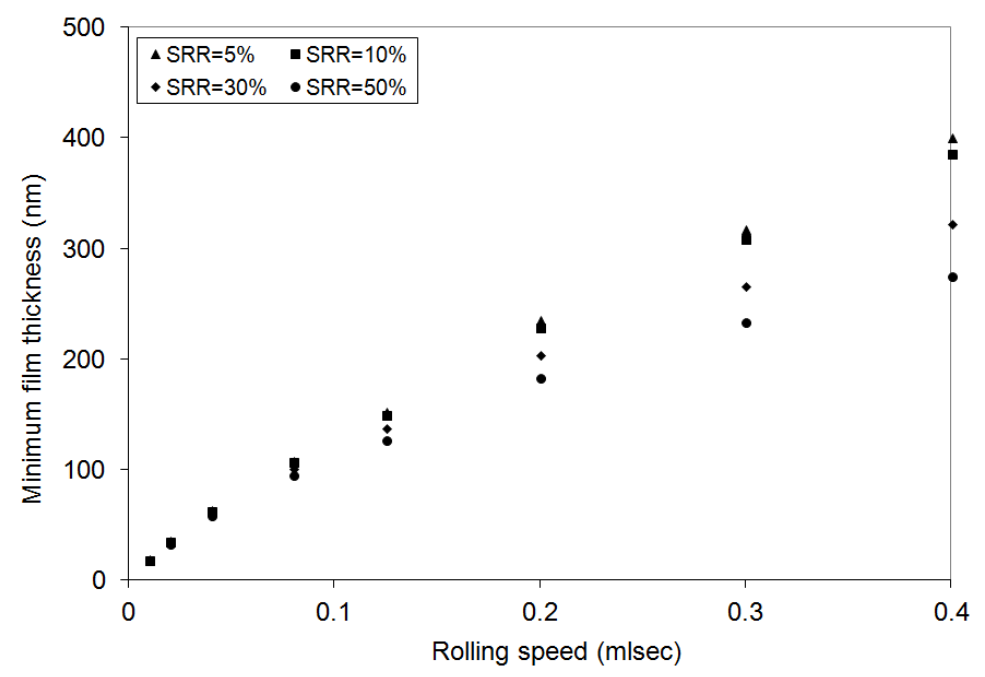

Figure 3. Minimum film thickness variation versus speed for different slide-roll ratio at constant load of $27 \mathrm{~N}$.

Figure 4 shows the pressure distribution and film thickness profiles in the direction of entraining motion through the central film for the Newtonian and nonNewtonian based solution using Ree-Eyring model for the different values of slide-roll ratio for speed of entraining motion of $0.4 \mathrm{~m} / \mathrm{sec}$ and an applied load of $27 \mathrm{~N}$. The main differences between the two models are that the film profile is not parallel through the contact region in the non-Newtonian solution as in the case of Newtonian solution. The second difference is that the pressure spike which is the characteristic of elastohydrodynamic lubrication is not clear in the case of non-Newtonian models in comparison to the case of Newtonian one. The same conclusion can also be shown by Huaiju Liu [9] and Ehret et al [32]. The reduction in film thickness as the slide-roll ratio increase is attributed to increase the value of the shear rate with increasing slide-roll ratio which leads to pronounced decreases in lubricant viscosity.

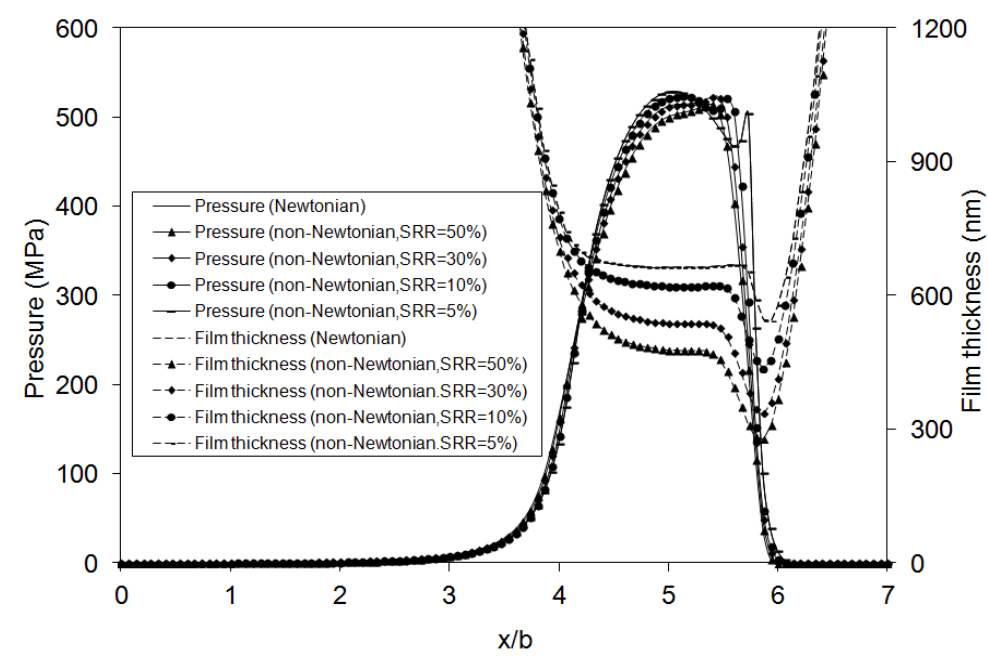

Figure 4. Pressure and film thickness profile for different slide-roll ratio for load of $27 \mathrm{~N}$ and speed of $0.4 \mathrm{~m} / \mathrm{sec}$. 
To see the characteristic of ultra-thin film for the case where non-Newtonian fluid using Ree-Eyring model is included, effects of surface liquid interaction of solvation and liquid-liquid interaction of Van der Waal's forces are considered in the numerical solutions in addition to the hydrodynamic action for the load balance equation to get the film thickness and pressure distribution shape. Table 3 shows the lubricants, material properties and the operating conditions used. In the current study, a non-polar lubricant of OMCTS was given between the two molecularly mica smooth surfaces as the separating media so the electrostatic force is not considered. The reason for choosing such molecularly smooth surfaces is that the solvation effects diminishes with rough surfaces under highly loaded conjunction as shown by Al-Samieh and Rahnejat [21].

Table 3. Lubricant, material properties and operating conditions

\begin{tabular}{lll}
\hline Designation & Properties & Values \\
\hline$\eta_{o}$ & Viscosity & $0.00235 \mathrm{~Pa} . \mathrm{s}$ \\
$\alpha$ & Viscosity coefficient for pressure & $10 \mathrm{GPa}^{-1}$ \\
$\varepsilon$ & Constant used in density equation (3) & $5.83 \times 10^{-10} \mathrm{~Pa}$ \\
$\xi$ & Constant used in density equation (3) & $1.68 \times 10^{-9} \mathrm{~Pa}$ \\
$\tau_{0}$ & Eyring model shear stress & $0.07 \mathrm{MPa}$ \\
$a$ & Molecular diameter & $1 \mathrm{~nm}$ \\
$A$ & Hamaker constant & $1.0 \times 10^{-19} \mathrm{Joule}$ \\
$C$ & Constant defined in equation (12) & $174 \mathrm{MPa}$ \\
$E$ & Reduced modulus of elasticity & $36 \mathrm{GPa}$ \\
$R_{x}$ & Reduced radius of curvature & $0.0127(\mathrm{~m})$ \\
$F$ & Load & $(0.02-20) \mathrm{mN}$ \\
$u$ & Speed & $0.0001 \mathrm{~m} / \mathrm{s}$ \\
\hline
\end{tabular}

Figure 5 shows minimum film thickness variation with applied load for the numerical solutions of Newtonian and non-Newtonian lubricant at speed of entraining motion of $100 \mu \mathrm{m} / \mathrm{sec}$. High value of slide-roll ratio (SRR) of $50 \%$ is used to show its effects on formation of film thickness and pressure distribution. In this figure the hydrodynamic non-Newtonian and Newtonian numerical results is shown for the purpose of comparison. The difference between the two models is that at high loads the film thickness is much smaller for the hydrodynamic non-Newtonian numerical solution than that predicted either from numerical solution based on Newtonian assumption or that predicted using either formulas of Brewe et al [33] or Hamrock and Dowson [34] for circular point contacts under iso-viscous rigid or iso-viscous elastic regimes of lubrication respectively. In facts, at high loads, the hydrodynamic pressure for nonNewtonian numerical solution can't support the applied load and cause the two contacting surfaces to be adhered together as the result of the decreased the viscosity of the lubricant. The physical explanation for this behavior is due to the reduction in the value of the coefficient of friction when the applied load is gradually increases. The same behavior has also been shown by Huaiju Liu [9]. Note that, for molecular level smoothness of surfaces such as used in the current numerical solution, the friction corresponds to formation of layers that cause a discretized form of the film thickness as the function of the applied load. Fortunately, this is not the case, because for ultra-thin film other forces such as liquid to solid and liquid to liquid interactions must be considered in supporting the applied load in addition to the hydrodynamic effects. 


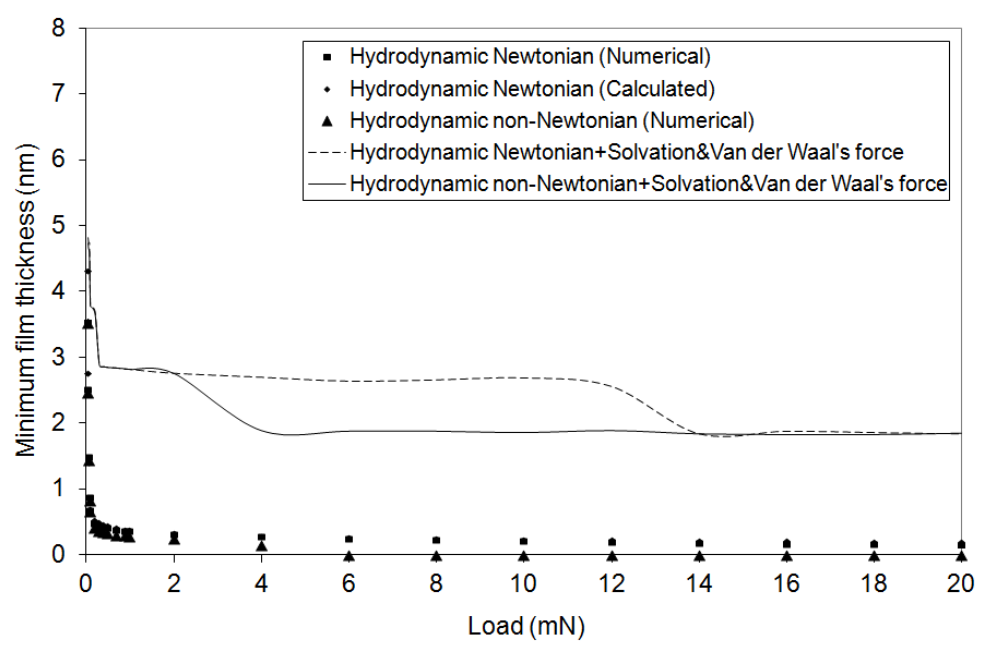

Figure 5. Minimum film thickness variation with load at constant speed of $100 \mu \mathrm{m} / \mathrm{sec}$ for Newtonian and non-Newtonian rheology under different effect of hydrodynamic and intermolecular force of solvation and Van der Waal's .

When the surface forces of solvation and Van der Waal's are aided to the hydrodynamic viscous action, the deviation of the non-Newtonian model from the Newtonian model starts at approximately high loads of about $2 \mathrm{mN}$ and consequently small film thickness results in lower value of the film thickness compared to that obtained using Newtonian model and the fluid film thickness are discretized at approximately gab intervals of $1 \mathrm{~nm}$ which equal to the molecular diameter of OMCTS similar to the solution of the Newtonian model (see Figure 5). In this case the net intermolecular forces of solvation and Van der Waal's in addition to the hydrodynamic action supports the load carrying capacity of the contact. Figure 6 supports the above discussion, where this figure shows the film thickness and pressure profiles in the direction of entraining motion through the central film for the case where the applied contact load of $12 \mathrm{mN}$ and speed of entraining motion of $100 \mu \mathrm{m} / \mathrm{sec}$ is used with the non-Newtonian rheology using Ree-Eyring model. From the shape of the pressure distribution shown in Figure 6, it is clear that the effect of the surface and fluid interaction which appears as an oscillation in the pressure shape.

An interesting feature appears in Figure 5 which is that the film thickness is never below than approximately $2 \mathrm{~nm}$ for both fluid models of Newtonian and nonNewtonian which is essential for the assumption of continuum mechanics for viscous flow in any conjunction as noted by Al-Samieh and Rahnejat $[20,21]$ and the continuum theory of Lifshitz that predicts the Van der Waal's force. The physical explanation for this feature is explained as follows; as the film thickness is reduced, effects of so-called solvation forces become very strong at short range and its value overcome the long range intermolecular force between the molecules of Van der Waal's, therefore, the net interaction of the solvation and Van der Waal's forces in addition to the hydrodynamic action including the lubricant rheology determined the magnitude of the adhesion between two contacting surfaces and keep two surfaces to be separated by the intervening media of hard spherical molecules of about $2 \mathrm{~nm}$ whatever the value of applied load.

Finally, minimum film thickness variation with respect to applied load at different Eyring shear stress is shown in Figure 7 for the case where intermolecular forces of solvation and Van der Waal's is included in the numerical analysis in addition 
to the hydrodynamic action. It is seen that, an increase in the value of Eyring shear stress is found to cause an insignificant enhancements in the values of minimum film thickness. This conclusion is contradicted to the results found by Huaiju Liu [9] and Manish Kaushik [35]. They showed that an increase in Eyring shear stress cause a substantial improvement in minimum and central film thickness for elastohydrodynmic lubrication analysis of line contacts because the viscosity increase as the Eyring shear stress increase. Therefore, from the results shown in Figure 7, it is clear that, a strong correlation can exist between the intermolecular force and the oil film thickness formation.

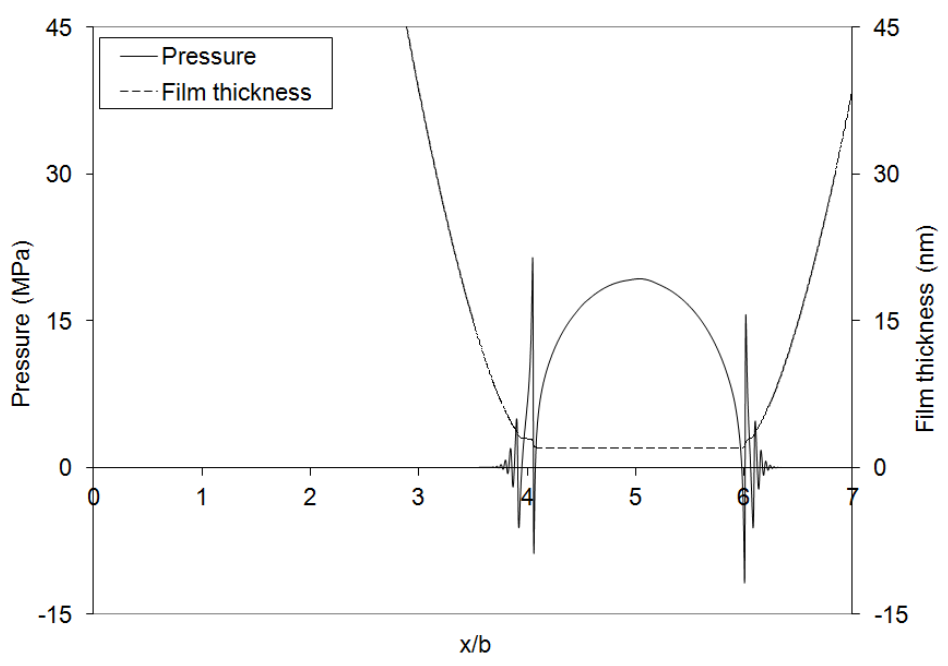

Figure 6. Pressure profile and film shape for non-Newtonian rheology in the central line of contact due to effect of hydrodynamic action and intermolecular force of solvation and Van der Waal's forces for applied load of $12 \mathrm{mN}$ and speed of $100 \mu \mathrm{m}$.

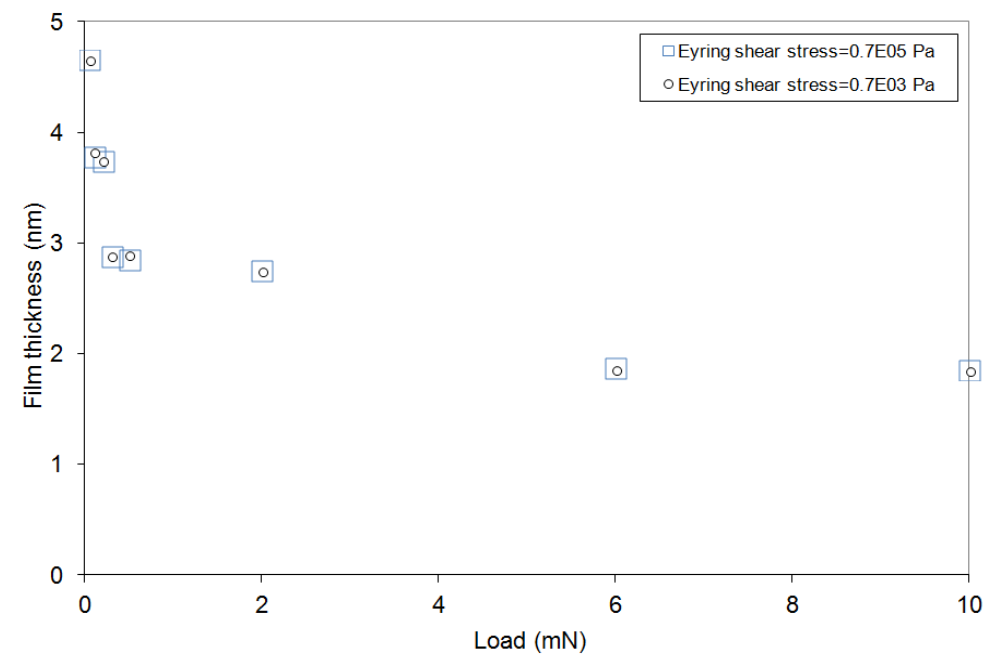

Figure 7. Variation of Eyring shear stress on film thickness versus load. 


\section{CONCLUSION}

A comparison between the Newtonian and non-Newtonian fluid using Ree-Eyring model is presented for elastohydrodynamic lubrication for point contact problem. The Reynolds equation incorporating the non-Newtonian fluid using Ree-Eyring model is used to show its effect on the formation of film thickness and pressure profile. It has been shown that, for the case where the hydrodynamic action is the only pressure acting to support the applied load capacity, the film thickness shape formed using nonNewtonian model is slightly different and its value is smaller than that formed using Newtonian case and pressure profile at the exit of the contact is different in that the pressure spike is lower than that shown for a Newtonian model. The main conclusion of this paper is that for ultra-thin film where the effect of intermolecular force of surfaceliquid interaction of solvation and liquid-liquid interaction of Van der Waal's are considered in addition to the hydrodynamic action, the film thickness formed using nonNewtonian model is smaller compared to that formed using Newtonian case. In this case, the discretization behavior of the film thickness and the oscillation nature of pressure profile occurred. The film thickness value is never below about a certain limit whatever the value of the applied load which is essential for the assumption of continuum mechanics for viscous flow and the continuum theory of Lifshitz. The results also showed that, for ultra-thin film, an insignificant enhancement in the minimum film thickness as the Eyring shear stress value increase.

\section{REFERENCES}

[1] Johnson K. Contact Mechanics. Cambridge: Cambridge University Press; 1985

[2] Gohar R. and Rahnejat H. Fundamentals of tribology. London: Imperial College Press; 2008.

[3] Sharma SC, Yadav SK. Performance analysis of a fully textured hybrid circular thrust pad bearing system operating with non-Newtonian lubricant. Tribology International, 2014; 77: 50-64.

[4] Mohammadpour M, Theodossiades S, Rahnejat H, Dowson D. Non-Newtonian mixed thermo-elastohydrodynamics of hypoid gear pairs. Journal of Engineering Tribology, Part J, 2018; 232: 1105-1125.

[5] Johnson KL, and Tevaarwerk JL. Shear behaviour of elastohydrodynamic oil films. Proceedings of The Royal Society A, 1977; 356: 215-236.

[6] Taha Sochi. Variational approach for the flow of Ree-Eyring and Casson fluids in pipes. International Journal of Modeling, Simulation, and Scientific Computing, 2016; 7: 1650007.

[7] Liu H, Mao K, Zhu C, Chen S, Xu X, Liu M. Spur gear lubrication analysis with dynamic loads. Proceedings of the Institution of Mechanical Engineers, Tribology Transactions, 2013; 56: 41-48.

[8] Lalit Vashishth, EHL analysis with actual ree-eyring model: National Institute of Technology; 2010.

[9] Huaiju Liu, Lubricated Contact Analysis of a Spur Gear Pair with Dynamic Loads: University of Warwick; 2013.

[10] Ildiko - Ficza, Petr Sperka and Martin Hartl, Transient calculations in elastohydrodynamically lubricated point contacts. Engineering Mechanics, 2014; 21(5): 311-319. 
[11] Ildiko' Ficza, Effects of non-newtonian lubricants on surface roughness in point contacts: Ústav konstruování; 2014.

[12] Spikes HA. Thin films in elastohydrodynamic lubrication: The contribution of experiment. Journal of Engineering Tribology, Part J, 1999; 213: 335-352.

[13] Guangteng G, Spikes HA. Boundary film formation by lubricant base fluids. Society of Tribologists and Lubrication Engineers, 1996; 39:448-454.

[14] Hartl M, Krupka I, Poliscuk R, Liska M, Molimard J, Querry M, Vergne P. Thin film colorimetric interferometry. Society of Tribologists and Lubrication Engineers, 2001; 44: 270-276.

[15] Lionel Bureau, Non-linear rheology of a nanoconfined simple fluid. Physical Review Letters, 2010; 104: 218302.

[16] Li-Ming Chu, Hsiang-Chen Hsu, Chia-Hsiang Su. Power law fluid model incorporated into thin film elastohydrodynamic lubrication of circular contacts. Transactions of the Canadian Society for Mechanical Engineering, 2015; 39: 547-556.

[17] Chan DYC, Horn RG. The drainage of thin liquid films between solid surfaces. The Journal of Chemical Physics, 1984; 83: 5311-5324.

18] Horn G, Israelachvili JN. Direct measurement of structural forces between two surfaces in a nonpolar liquid. The Journal of Chemical Physics, 1981; 75 (3): 1400-1411.

[19] Israelachvili JN. Intermolecular and surface forces. New York: Academic Press; 2010.

[20] Al-Samieh MF, Rahnejat H. Nano-lubricant film formation due to combined elastohydrodynamic and surface force action under isothermal conditions. Proceedings of the Institution of Mechanical Engineers, 2001; 215: 1019-1029.

[21] Al-Samieh MF, Rahnejat H. Ultra-thin lubricating films under transient conditions. Journal of Physics D: Applied Physics, 2001; 34: 2610-2621.

[22] Chong WWF, Teodorescu M, Rahnejat H. Effect of lubricant molecular rheology on formation and shear of ultra-thin surface films. Journal of Physics D: Applied Physics. 2011; 44(16): 165302.

[23] Ku ISY, Chong WWF, Reddyhoff T, Rahnejat H. Frictional characteristics of molecular length ultra-thin boundary adsorbed films. Meccanica, 2015; 50(7): 1915-1922.

[24] Al-Samieh MF. Effect of Changing Ellipiticity Ratio on the Formation of UltraThin Lubricating Film. Tribology in Industry, 2017; 39(4): 431-443.

[25] Al-Samieh MF. Film thickness formation in nanoscale due to effects of Elastohydrodynamic, Electrostatic and Surface force of Solvation and Van der Waals. Industrial Lubrication and Tribology, 2018; 70: 51-58.

[26] Greenwood JA. Two-dimensional flow of a non-Newtonian lubricant. Proceedings of the Institution of Mechanical Engineers Part J-Journal of Engineering Tribology, 2000; 214: 29-41

[27] Canova, Lutz H Gade, Thomas A Jung and Ernst Meyer. Van der Waals interactions and the limits of isolated atom models at interfaces. Nature Communications, 2016; 7: 1-7.

[28] Thaicharoen N, Schwarzkopf A, Raithel G. Measurement of van-der-Waals interaction by atom trajectory imaging. Physical Review A, 2015; 92 (6): 1-6.

[29] Prakash Chandra Mishra. Effect of Surface Forces on Ultrathin Film Lubrication. ISRN Tribology, 2014; 2014 (1): 1-9. 
[30] Morciano M, Fasano M, Nold A, Braga CC, Yatsyshin P, Sibley DN, Goddard BD, Chiavazzo E, Asinari P, Kalliadasis S. Nonequilibrium molecular dynamics simulations of nanoconfined fluids at solid-liquid interfaces. Journal of Chemical Physics, 2017; 146 (24): 1-13.

[31] Peiyuan Sun, Meirong Zhao, Jile Jiang, Yelong Zheng, Yaqian Han and Le Song. The Differential Method for Force Measurement Based on Electrostatic Force. Journal of Sensors, 2017; 2017 (1):1-7

[32] Ehret P, Dowson D, Taylor CM. On lubricant transport conditions in elastohydrodynamic conjunctions. Proceedings of the Royal Society A, 1998; 454: 763-787

[33] Brewe DE, Hamrock BJ, Taylor CM. Effect of geometry on hydrodynamic film thickness. Journal of Tribology, 1979; 101: 231-239.

[34] Hamrock BJ, Dowson D. Elastohydrodynamic lubrication of elliptical contacts for materials of low elastic modulus. Part I- Fully flooded conjunction. Journal of Tribology. 1978; 100 (2): 236-245.

[35] Kaushik M. Elastohydrodynamic lubrication analysis using electro-rheological fluids under dynamic loads: National Institute of Technology; 2015. 